\title{
Numerical Analysis of Multiplication layer for InGaAs/InP Single Photon Avalanche Diodes
}

\author{
Q. Y.Zeng, W. J. Wang*,W. D. Hu,N. Li, W. Lu* \\ National Laboratory for Infrared Physics,Shanghai Institute of Technical Physics,Chinese Academy of Sciences,500 Yu Tian \\ Road,Shanghai,200083,China \\ wangwi@mail.sitp.ac.cn, luwei@mail.sitp.ac.cn
}

\begin{abstract}
In this paper, we theoretically study the electrical properties of a separate absorption, grading, charge,and multiplication InGaAs/InP avalanche photodiodes(APD) on the multiplication layer for different carrier lifetime,doping and traps concentration. These characteristics can be used to analyze some problems in the process of device fabrication.
\end{abstract}

\section{I . INTRODUCTION}

In recent years, avalanche photodiodes(APD) have attracted more and more attention since there can be used in Geiger mode for single photon detection [1]. Low dark current is an important prerequisite for single photon detection. There were many works about theoretical study of APDs and single photon avalanche diodes(SPADs) [2]-[5], however, most papers just concerned about the structure parameters, such as the thickness of the multiplication layer, the doping of the charge layer $[4,5]$. After the actual device fabrication, APDs with similar structure parameters often show much difference in performance, which may be influenced by the fabrication process, such as introducing the traps.

During actual fabrication, $\mathrm{p}$ doping of the device is formed by zinc diffusion, which will influence the multiplication layer doping concentration or the $\mathrm{p}$ doping layer and multiplication layer interface. In this paper, effects of the carrier lifetime, the doping concentration and traps concentration of the multiplication to the APD's performance are discussed. These theoretical results will be instructive for the device fabrication.

\section{MODEL DESCRIPTION AND NUMERICAL RESULTS}

The basic structure of the separate absorption, grading, charge, and multiplication(SAGCM) InGaAs/InP APD under consideration is shown in Fig. 1. Numerically simulation of the device is performed using the software ISE-TCAD, with the carrier generation-recombination process accounting for Shockley-Read-Hall, Trap-Assisted Tunneling(TAT), Auger, Radiative,Band-to-Band Tunneling mechanisms.

\section{(a) Doping concentration}

The multiplication layer is intrinsic in theory, but the $\mathrm{Zn}$ concentration of the $\mathrm{p}+\operatorname{InP}$ layer is an error function distribution in the thermal diffusion process[7], which is equivalent to changing the dopant concentration of the multiplication layer,or even introducing traps to the interface between multiplication layer and $\mathrm{p}+\mathrm{InP}$ layer. We change the doping concentration of multiplication layer from $1 \mathrm{E} 15 \mathrm{~cm}^{-3}$ to $4 \mathrm{E} 16$

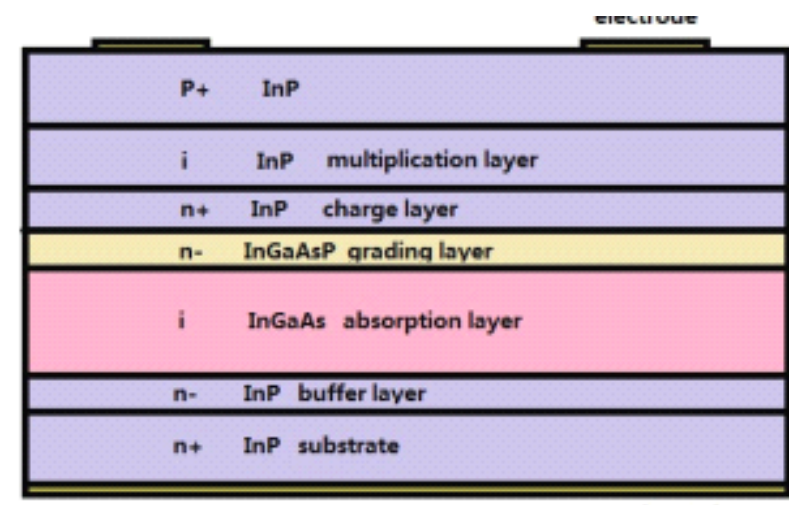

Fig. 1. Schematic cross section of the simulated APD

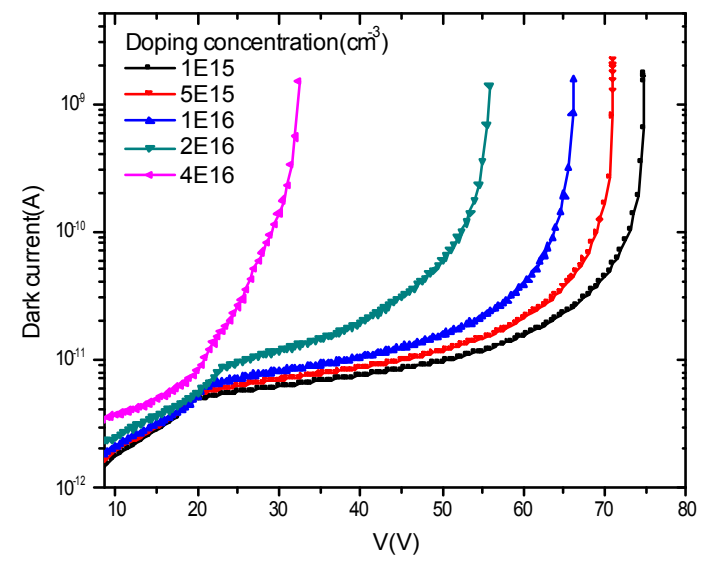

Fig. 2 Dark current versus different doping concentration

$\mathrm{cm}^{-3}$, as seen in Fig. 2. It is shown that the breakdown voltage decrease linearly (from $75 \mathrm{~V}$ to $33 \mathrm{~V}, 0.9 \mathrm{~V} / 1 \mathrm{E} 15 \mathrm{~cm}^{-3}$, as seen in Fig. 3) with the increase of the doping concentration, but the dark current at $0.95 \mathrm{~V}_{\mathrm{b}}$ are similar $(60 \mathrm{pA})$.

(b) Carriers lifetime

The carriers lifetime of the InGaAs absorption layer and InGaAsP grading layers are set to $2.2 \mathrm{E}-4 \mathrm{~s}$ since these layers are intrinsic or lightly doped, and InP substrate layer, charge layer and $\mathrm{p}+\mathrm{InP}$ layer are set to $1.0 \mathrm{E}-9 \mathrm{~s}$ due to heavily doped. The carrier lifetime of multiplication layer varies from $1 \mathrm{E}-7 \mathrm{~s}$ to $1 \mathrm{E}-10 \mathrm{~s}$. As seen in Fig. 4, carriers lifetime only affects the dark current linearly, which illustrate that the carriers lifetime only relevant to the generation-recombination current.

(c) Traps concentration 


\section{NUSOD 2013}

We simulated the I-V characteristics with different traps concentration in multiplication layer, which is shown in Fig. 5. The breakdown voltage decreased from $75 \mathrm{~V}$ to $67 \mathrm{~V}$, and the dark current at $0.95 \mathrm{Vb}$ increased from $50 \mathrm{pA}$ to $2 \mathrm{nA}$ when the traps concentration increased from $1 \mathrm{E} 14 \mathrm{~cm}^{-3}$ to $4 \mathrm{E} 16 \mathrm{~cm}^{-3}$. Why these happened may owe to the trap-assisted tunneling process and generation-recombination centers. On one hand, the trap-assisted tunneling increase the dark current, especially in the region prior to breakdown, for example,region at $0.95 \mathrm{Vb}$. On the other hand, traps are the generation-recombination centers which can reduce the carriers lifetime and increase the carrier concentration, and lead to dark current increasing and breakdown voltage decreasing.

Another phenomenon can also prove this interpretation. The traps with same concentration but with different energy levels show different results in the dark current and breakdown voltage. For example,the dark current at $0.95 \mathrm{~V}_{\mathrm{b}}$ shown no change with the traps concentration when the traps energy were set at $0.04 \mathrm{eV}$ below the conduction band. These traps have no as sis tance to the tunneling current.

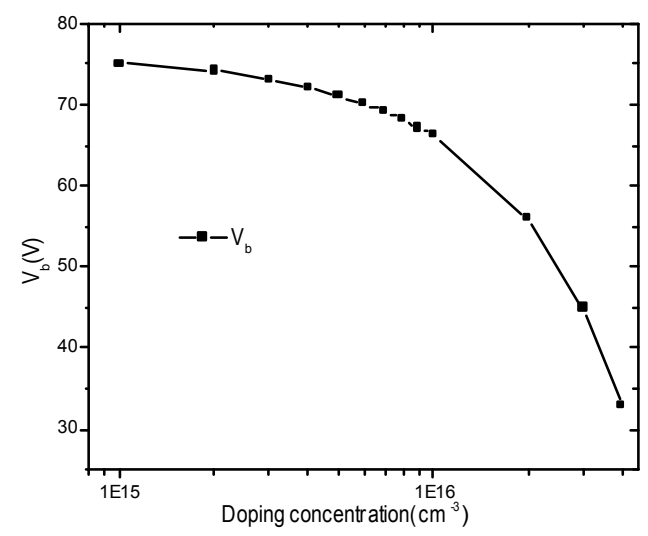

Fig. 3 breakdown voltage versus doping concentration

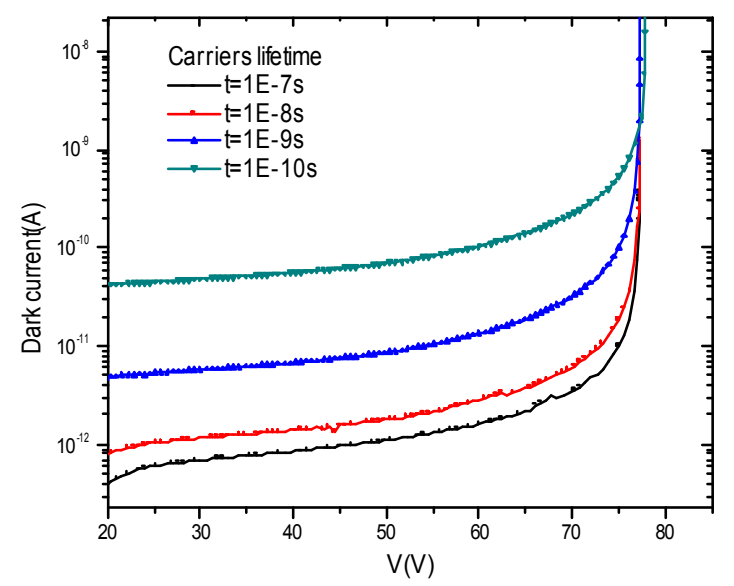

Fig. 4 Dark current versus different carriers lifetime

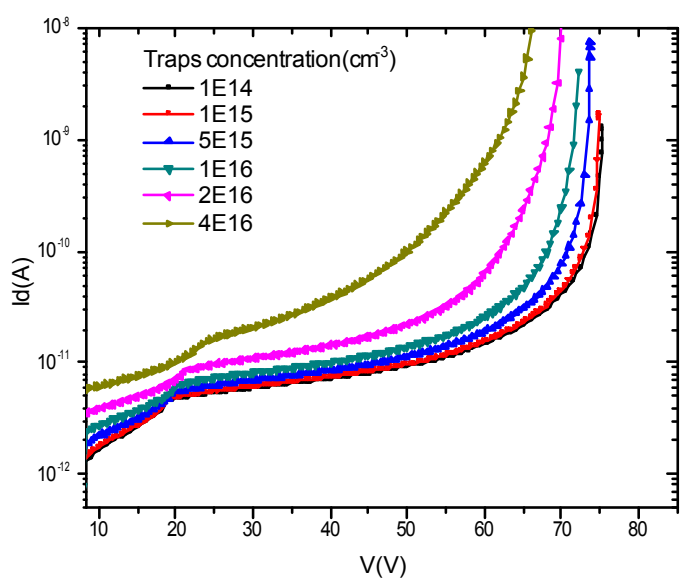

Fig. 5 Dark current versus different traps concentration

\section{CONCLUSION}

We have performed a theoretical study of the multiplication layer characteristics of the SAGCM InGaAs/InP APD. The carriers lifetime, doping concentration and traps affect the dark current and breakdown voltage with different degrees, which are instructive for the fabrication process and helpful to improve the single photon detection performance.

\section{ACKNOWLEDGMENT}

This work was supported by Strategic Pilot Program of Science and Technology of Chinese Academy of Sciences (XDB01010200), National Research Program of MOST (2011CB925600) and National Natural Science Foundation of China (08K0510W15).

\section{REFERENCES}

[1] K.A. Patel,J.F. Dynes,A.W.Sharpe,Z.L.Yuan,R.V.Penty,A.J.Shields. "Gigacount/second Photon Detection with InGaAs Avalanche Photodiodes", Electronics Letters, vol. 48,pp.111-113,2012

[2] S. Pellegrini, R. E. Warburton, L. J. J. Tan et al. "Desing and Performance of an InGaAs-InP Single-Photon Avalanche Diode Detector,' IEEE J. Quantum Electron, vol.42, №. 4, pp. 397-403, 2006.

[3] K. Sugihara, E. Yagyu, Y. Tokuda."Numerical Analysis of Single Photon Detection Avalanche Photodiodes Operated in the Geiger Mode" J. Appl. Phys:99,124502,2006

[4] L. Lin, W. J. Wang, N. Li, W. Lu."Theoretical Study of Separate Absorption, Grading, Charge, and Multiplication InGaAs/InP Single Photon Avalanche Diode".NUSOD'08. International Conference,pp. 83-84,2008

[5] W. J. Wang, L. Lin, T. X. Li,N. Li, W. D. Hu, W. Lu,X, S. Chen. "Numerical Analysis of Single Photon Avalanche Photodiodes with Improved Structure".NUSOD'10. International Conference, pp. 19-20,2010

[6] R. J.McINTYRE,"Multiplication Noise in Uniform Avalanche Diodes", IEEE Trans. Electron Devices, vol.13,No.1,pp.165,1966

[7] B. Tuck ,A. Hooper," Diffusion Profiles of Zinc in Indium Phosphide", J. Phys. D:Appl. Phys.,vol. 8,pp.1808,1975 